\title{
Dosimetry and spectral analysis of a radiobiological experiment using laser-driven proton beams
}

\author{
F. Fiorini ${ }^{a}$, D. Kirby ${ }^{a}$, M. Borghesi ${ }^{b}$, D. Doria ${ }^{b}$, J.C.G. Jeynes ${ }^{c}$, \\ K.F. Kakolee ${ }^{b}$, S. Kar ${ }^{b}$, S.K. Litt ${ }^{b}$, K.J. Kirkby ${ }^{c}$, \\ M.J. Merchant ${ }^{c}$ and S. Green ${ }^{d}$ \\ ${ }^{a}$ School of Physics and Astronomy, University of Birmingham, Edgbaston, \\ Birmingham, B15 2TT, UK \\ ${ }^{b}$ Department of Physics and Astronomy, Queen's University of Belfast, Belfast, \\ BT7 1NN, UK \\ ${ }^{c}$ Ion Beam Centre, University of Surrey, Stag Hill, Guildford, GU2 7XH, UK \\ ${ }^{d}$ Hall-Edwards Radiotherapy Research Group, Department of Medical Physics, \\ University Hospital Birmingham NHS Trust, Birmingham B15 2TH, UK \\ E-mail: fxf817@bham.ac.uk
}

\begin{abstract}
Laser-driven proton and ion acceleration is an area of increasing research interest given the recent development of short pulse-high intensity lasers. Several groups have reported experiments to understand whether a laser-driven beam can be applied for radiobiological purposes and in each of these the method to obtain dose and spectral analysis was slightly different. The difficulty with these studies is that the very large instantaneous dose rate is a challenge for commonly used dosimetry techniques, so that other more sophisticated procedures need to be explored. This article aims to explain a method for obtaining the energetic spectrum and the dose of a laser-driven proton beam irradiating a cell dish used for radiobiology studies. The procedure includes the use of a magnet to have charge and energy separation of the laser driven beam, Gafchromic films to have information on dose and partially on energy, and a Monte Carlo code to expand the measured data in order to obtain specific details of the proton spectrum on the cells. Two specific correction factors have to be calculated: one to take in account the variation of dose response of the films as a function of the proton energy and the other to obtain the dose to the cell layer starting from the dose measured on the films.

This method, particularly suited to irradiation delivered in a single laser shot, can be applied in any other radiobiological experiment performed with laser driven proton beams, with the only condition that the initial proton spectrum has to be at least roughly known.

The method was tested in an experiment conducted at Queen's University of Belfast using the TARANIS laser, where the mean energy of the protons crossing the cells was between 0.9 and $5 \mathrm{MeV}$, the instantaneous dose rate was estimated to be close to $10^{9} \mathrm{~Gy} / \mathrm{s}$ and doses between 0.8-5 Gy were delivered to the cells in a single laser shot. The combination of the applied corrections modified the initial estimate of dose by up to $40 \%$
\end{abstract}




\section{Introduction}

Today proton/ion therapy has become one of the most successful treatments to cure cancer, but due to the high costs and sizes of the facilities, it has not easily spread worldwide. Recently, laser driven ion facilities are becoming a promising alternative because even if the technology is still nascent and needs several improvements, it has been estimated that costs, complexity and sizes of an entire facility would be considerably reduced $[12,2]$. Laser Induced Beams of Radiation and their Applications (LIBRA) is a UK consortium which aims to develop a new type of ion source by shining an ultra intense laser beam onto a small target of metal, plastic, liquid or gas. One of its proposed applications is ion beam radiation therapy for cancer treatment.

In a typical laser-ion acceleration experiment, a high power laser pulse (of intensity above $10^{19} \mathrm{~W} / \mathrm{cm}^{2}$ ) is focused onto a thin foil. A prepulse, due to the laser amplification system and less energetic than the main pulse, creates the plasma on the surface of the target. When the main pulse arrives, it interacts preferentially with this plasma and a population of hot electrons with a Maxwellian-type distribution is generated [17]. These electrons traverse the target and build up a high electrostatic field, up to the order of $\mathrm{TV} / \mathrm{m}$, capable of accelerating the particles on the rear surface of the foil [10]. In addition to the acceleration of some heavy ion constituents of the target, hydrocarbons and water contaminants on the surface of the target are also ionised and accelerated [3]. Due to their higher charge-to-mass ratio, protons are more efficiently accelerated than other ion species, reducing the effectiveness of the approach for heavier ion acceleration. The produced proton/ion beams accelerated by this mechanism (called Target Normal Sheath Acceleration - TNSA) exhibit advantageous characteristics, such as short pulse lengths, high currents and low transverse emittance, but they also show exponential energy spectra with almost 100\% energy spread. So far, for proton beams the maximum energy that has been achieved is $\sim 60 \mathrm{MeV}[3]$ and for ions (typically carbon and oxygen) $\sim 10 \mathrm{MeV} / \mathrm{n}$ [11]. The large energy spread and the relatively low maximum energy remain the significant impediments for this technique to be applied for medical purposes.

While many efforts are employed to improve the characteristics of the laser driven beams to adapt them to the requirements of a cancer therapy treatment, so far, only a few studies have been carried out to understand whether the high dose rate of these beams might cause different biological consequences from the well known effects of conventionally accelerated ion beams $[19,9]$. In these previous experiments, the total dose on the cells was obtained by using multiple laser shots.

The experiment described in this article has been conducted using the Terawatt Apparatus for Relativistic and Nonlinear Interdisciplinary Science (TARANIS) laser at Queen's University of Belfast [13], but the method can be applied to any other high power laser-driven ion accelerator. The difficulty with these kind of experiments is that the large instantaneous dose rate is a challenge for commonly used dosimetry techniques, so that other procedures need to be explored and improved $[8,18,5]$. 
Dosimetry and spectral analysis of a radiobiological experiment using laser-driven proton beams 3

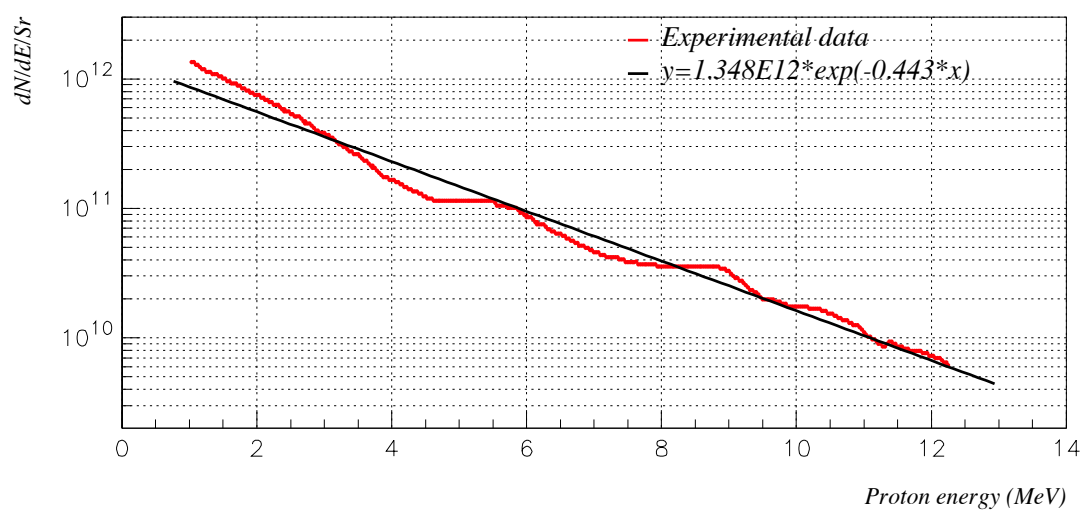

Figure 1. Typical spectrum of the proton beam accelerated by the TARANIS laser.

Through the TNSA mechanism a beam of protons (but also photons and electrons) with the typical broad spectrum shown in figure 1 was produced. The acceleration of heavy ions via TNSA from TARANIS or any other laser with similar characteristics is very inefficient [6]. The accelerated particles were made to pass through a collimator and a magnet. Part of the proton beam was then directed onto a dish containing V79 chinese hamster cells and to the Gafchromic films. A preliminary example of cell survival graph, where each dose point was obtained from a single laser shot, is shown at the end of the paper.

In this article we will focus mainly on the method used to obtain the spectral analysis of the proton beam interacting with the cells and the extraction of the deposited dose, rather than on the radiobiology details.

\section{Method}

\subsection{Overall set-up of the experiment}

The overall experimental setup is illustrated in figure 2 (a). In it the red beam represents the TARANIS laser (nominal characteristics: up to $20 \mathrm{~J}$ but during the experiment $5 \mathrm{~J}, 500 \mathrm{fs}$ at $1053 \mathrm{~nm}$ wavelength) which was focused onto a $12 \mu \mathrm{m}$ thick aluminium target at intensities of the order of $10^{19} \mathrm{Wcm}^{-2}$. As is typical of these experimental conditions, predominantly protons are accelerated, with the characteristic spectrum shown in figure 1. A collimator (500 $\mu \mathrm{m}$ of aperture) was used to select only the low divergence particles and, also, to maintain as low as possible the irradiation time. Subsequently, a dipole magnet of $0.9 \mathrm{~T}$, was used both to discriminate between accelerated electrons and protons and to partially resolve the proton energy spectrum. At a distance of $14 \mathrm{~cm}$ from the magnet, a $50 \mu \mathrm{m}$ thick mylar foil was used as a chamber window. The dish, containing some medium and a cell monolayer, and the films, were placed vertically in air and parallel to this window (see figure 2 (b)). The angle between the normal to the vertical face of the magnet and the normal to the window could be varied to select the energy of the protons which perpendicularly irradiated the dish. 


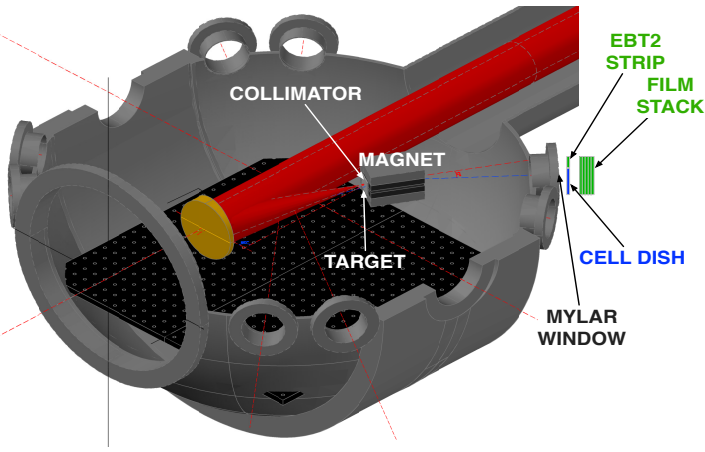

(a)

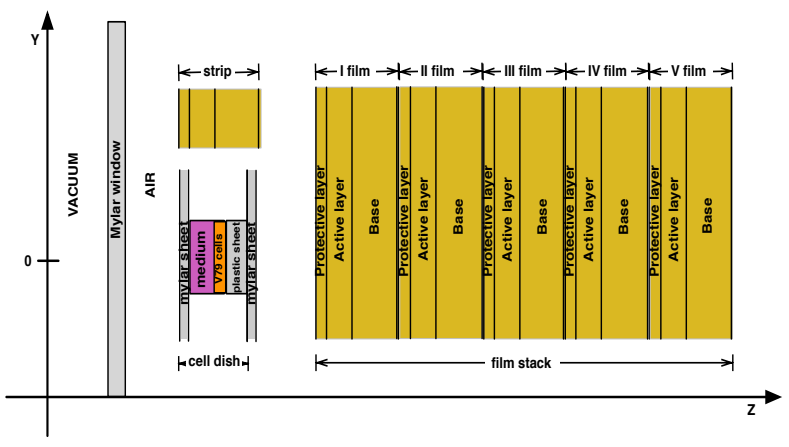

(b)

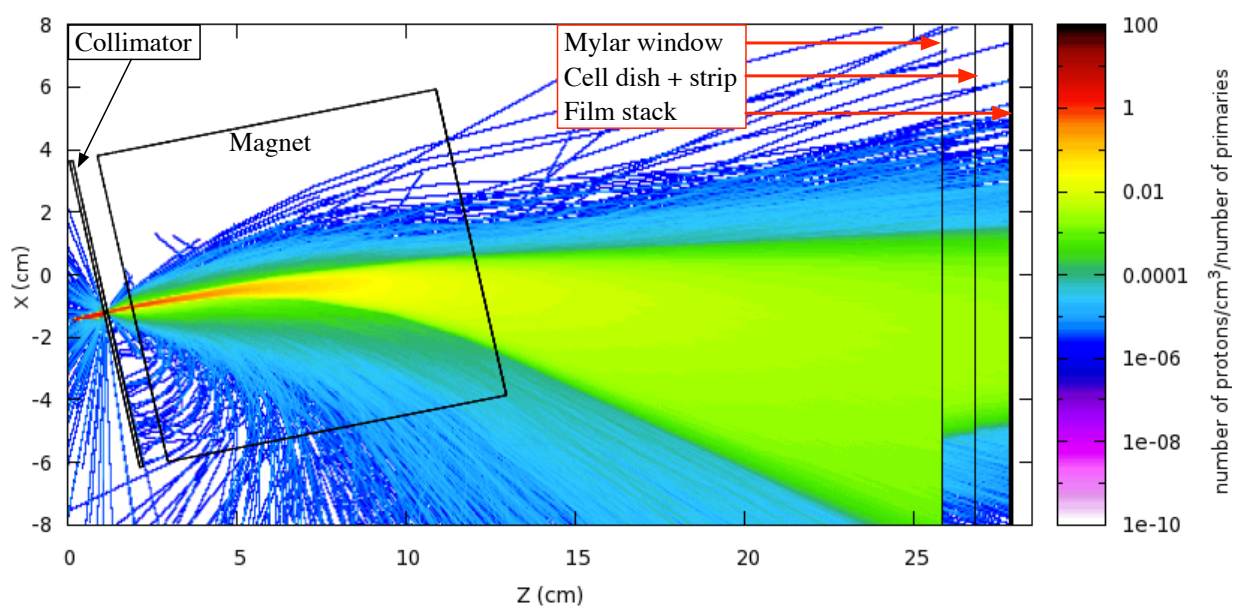

(c)

Figure 2. (a) Section of the experimental setup. (b) Schematic representation on Y-Z plane of the cell dish and film stack, not in scale. (c) Geometry with simulated proton tracks on X-Z plane: only the protons with an energy higher than $2 \mathrm{MeV}$ can reach the cell dish.

All the figures in this paper refer to the configuration where that angle was fixed at 12 degrees, which allowed $\sim 6 \mathrm{MeV}$ protons to perpendicularly strike the dish and the films. Another configuration was also used to allow $~ 3.6 \mathrm{MeV}$ protons to perpendicularly strike the cell dish. Moreover, having used a magnet the electrons and the X-rays accelerated by the laser could not reach the cells: the electrons being deviated in the opposite direction and the X-rays continuing straight.

The V79 cells were left to grow on a thick plastic foil which at the time of irradiation was cut in $3 \mathrm{~mm}$ diameter circles. Then, the cell dish was made inserting these circles between two $3 \mu \mathrm{m}$ thick mylar foils. For the majority of the cases the average thickness of the cell monolayer and some liquid medium to keep the cells moist was $(34 \pm 5) \mu \mathrm{m}$

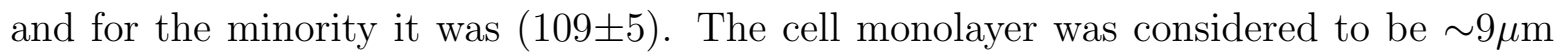
thick as described in [15] and confirmed by our measurements.

The challenge for dosimetry is to determine the dose to cells for each laser shot. To achieve this, a stack of films was placed $1 \mathrm{~cm}$ behind the cell dish for all shots. An 
additional film strip was placed at the same distance from the window as the cells in order to measure the deposited dose on the cells in the most accurate way possible. In figure 2 (b), the film stack and the strip are shown in yellow, the cells in orange and the medium in pink.

\subsection{Estimate of the dose in the cell spots}

The setup, from the proton source to the film stack, has been simulated using the Monte Carlo code Fluka $[1,4]$. Distances and angles were modelled to be, as far as possible, equal to the experimental ones. The initial proton beam had the exponential spectrum represented by the fit of the curve in figure 1 . The simulated proton tracks are shown in figure 2 (c). The very low energetic protons were stopped by the mylar window (represented by the first vertical line at $\sim 26 \mathrm{~cm}$ from the origin), but those with an initial energy higher than $2 \mathrm{MeV}$ could reach the dish and the strip (represented by the second line) and then the film stack (represented by the final thicker line). The Gafchromic films used are a special unlaminated edition of the standard EBT2 films (International Specialty Products, Wayne, NJ, USA). These special EBT2 films (lot number: A10150902), not commercially available, present the advantage of having the active layer closer to one of the surfaces: $5 \mu \mathrm{m}$ of top-coat layer, $30 \mu \mathrm{m}$ of active layer and $175 \mu \mathrm{m}$ of polyester substrate. The normal EBT2 films would have been very thick for these low proton energies and possibly a part of information about dose and beam energy would have been lost in the non active thickness. The films were previously calibrated using the $29 \mathrm{MeV}$ beam accelerated by the cyclotron of the University of Birmingham, for doses up to 14 Gy (procedure explained in [7]). The equation of calibration is given in equation 1, where D indicates the dose to water in Gy, OD the optical density and GV the grey value of the pixels of the red channel scan of the film:

$$
\mathrm{D}(\mathrm{Gy})=\mathrm{e}^{(\mathrm{a}+\mathrm{bOD})}+\mathrm{cOD}+\mathrm{d} \text { where } \mathrm{OD}=-\log (\mathrm{GV} / 65535)
$$

From the calibration the parameters are: $\mathrm{a}=(0.56 \pm 0.03), \mathrm{b}=(2.70 \pm 0.03), \mathrm{c}=(-4.9 \pm$ $0.3), \mathrm{d}=(-2.4 \pm 0.1)$.

An example of irradiated films in a laser shot is shown in figure 3 (a). The deposited dose on the strip and on the first film of the stack decreases from the left to the right: the maximum dose corresponds to the lowest energy protons which deposit all their energy in the film. The minimum dose corresponds to the highest energy protons, which loosing energy in the films, were stopped in the last film of the stack. The protons with an initial energy higher than 4.85 MeV, 6.61 MeV, 8.13 MeV and 9.4 MeV could reach respectively the second, third, fourth and fifth film. A sixth film (here not shown) would be darkened by protons having a minimum energy of $10.5 \mathrm{MeV}$. Since nothing was observed on the sixth film with any of the used configurations, we conclude that the maximum energy of the protons irradiating the cell dish was between 9.4 and $10.5 \mathrm{MeV}$. As can be seen in figure 3 (a) during this irradiation there were no cells in the dish.

An example of the red channel scan of the films used during a cell irradiation is shown in figure 3 (b). The lighter areas are the projections of the cell spots. With this 
Dosimetry and spectral analysis of a radiobiological experiment using laser-driven proton beams6
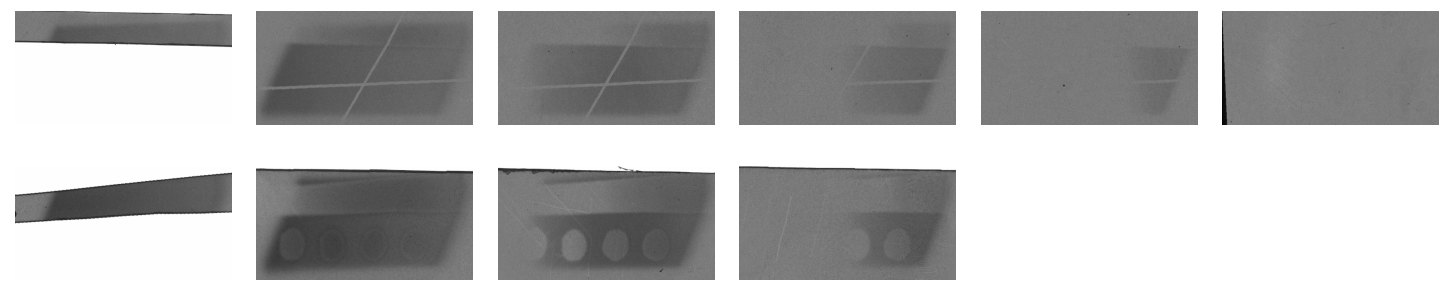

Figure 3. Red channel of the scan of a stack (a) used during no cell irradiation and another one (b) used during cell irradiation. The lighter areas are the cell spot projections indicated from left to right as A, B, C and D.

method it was possible to irradiate up to four cell spots (indicated from left to right as $\mathrm{A}, \mathrm{B}, \mathrm{C}$ and $\mathrm{D}$ ), however the geometry of the vacuum chamber window meant that the optimal number was three (B, C and D). After having aligned all the films of the same stack using the TurboReg plugin [16] of ImageJ [14], the real cell locations have been evaluated for each shot considering both the projections and the position of the cross hairs (placed immediately behind the empty cell dish) which was used as a reference. For both the situations the shape of the outline of the beam on the films was due to the oblique shape of the chamber window.

Knowing the original positions of the cell circles and as there was no appreciable variation in energy or dose along the vertical axis, the first estimates of the doses $\left(d_{i j}\right.$, where $i$ is the shot and $j$ the cell spot) were calculated using the average of the grey values of the pixels corresponding to the cell spots on the strip (see figure 4) and the calibration in equation 1 . For the cell spot $\mathrm{A}$ the dose cannot be measured, because part of the spot was outside the beam window on the strip. This is the first step in calculating the dose: two corrections must then be made. One is due to the variation of the dose response of the films with proton energy as reported in [8], and the other is from the fact that the dose to water was measured on the strip and not on the cell layer which had a slightly different thickness and position. The first correction requires the spectrum of the protons hitting the active layer of the strip to be known and it has a particular effect on the doses due to the protons at very low energy $\left(\mathrm{E}_{p}<4 \mathrm{MeV}\right)$ as explained below. The second depends entirely on the position and on the thicknesses of the active layer of the strip and of the cell layer. In fact, using the above mentioned calibration giving directly the dose to water, the only difference between the doses we obtain after the first correction and the doses actually absorbed by the cells is due to the position and the thickness of the materials which the protons have to cross. In order to calculate both the corrections simulations of the experiment were indispensable.

\subsection{Determination of the correction due to the dose response of the films}

In the first part of this calculation the cell dish was simulated without cells, medium and plastic foil, but taking into account only the two thin mylar foils and the air between them. The reason for this approach is that the simulation of the films of the stack gives the geometric relation between the simulated and the experimental reference systems, 


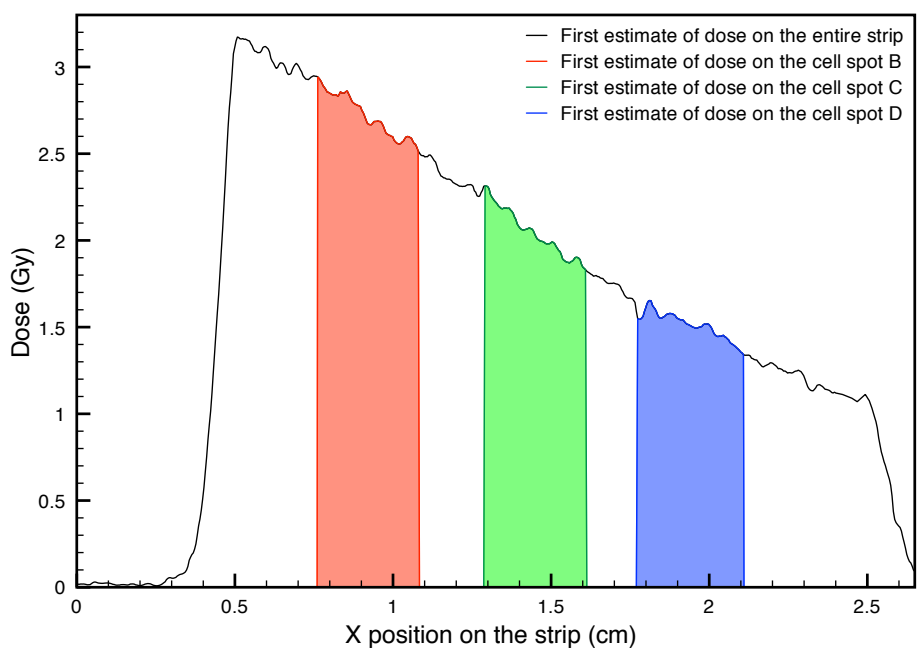

Figure 4. Graph of the dose along the strip in figure 3 (b): the filled regions represent the doses related to the cell spot B (in red), C (in green) and D (in blue). For the cell spot A the dose cannot be measured on the strip because of the chamber window shape.

experimentally measured on the films in the region where the cells were absent (darker regions of figure $3(\mathrm{~b})$ ).

By measuring the position of the dose edges on each experimental and simulated films it was possible to relate the simulations to the experimental results. Figure 5 is an example of simulated protons hitting the stack: in black are the positions of the protons crossing the active layer of the first film, in red the positions of the protons crossing the active layer of the second film and so on up to the fifth film.

From the irradiated films like those in figure 3 (b) and from figure 5, it was possible to compare the distance between two different dose edges on the experimental films and on the simulated ones and find the translation value between the reference system of the experiment and of the simulation for each shot. The dose edge distances on the films were determined by the distances between the points where two different films of the same stack start darkening, except for the first film which is always dark in all its length for all the used configurations. The dose edge distances on the simulated films were obtained by the distances between the beginnings of two different colour regions, excluding the black one corresponding to the first film. If these distances are in good agreement, we used a tolerance of $\pm 5 \%$, it can be assumed that the simulated case is representative of the experimental shot. If, in a shot, there is a disagreement between the distances of two dose edges in the simulation and in the experimental stack, or, the dose distribution on the strip is not as regular as in figure 4, it means that the initial proton spectrum is significantly changed because of unknown factors. In these cases, obtaining the energy on the cells from the simulation is not possible. For this reason, it is preferable to use, for each shot, as many films as possible in the stack: the suggested maximum number is determined by the number of films which would be darkened by 
Dosimetry and spectral analysis of a radiobiological experiment using laser-driven proton beams8

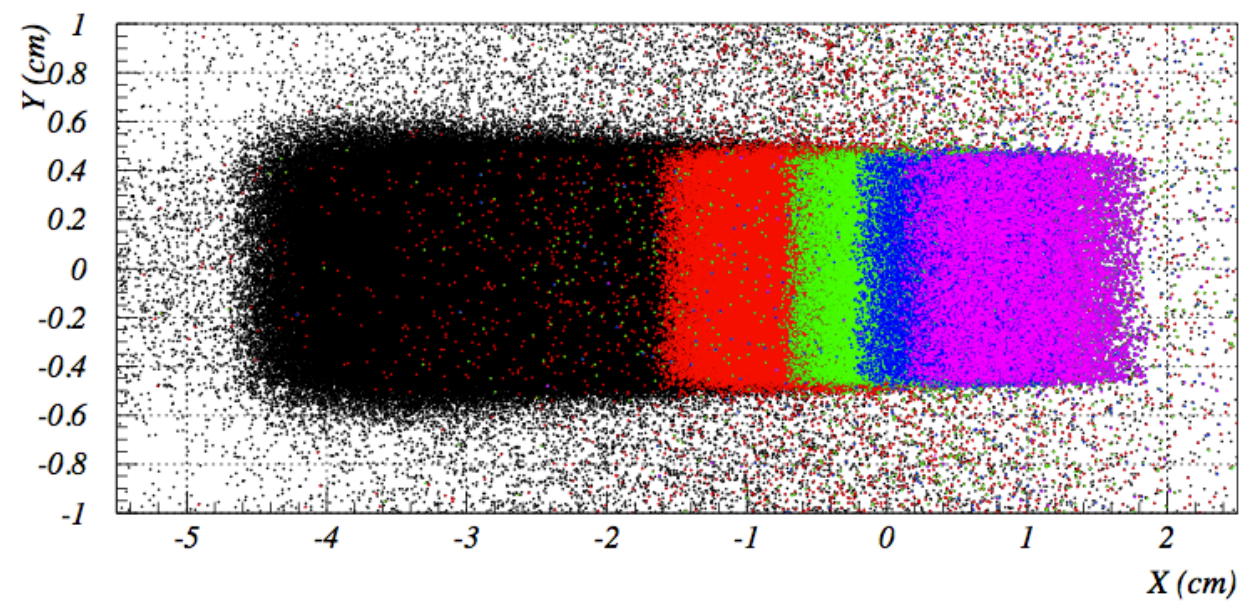

Figure 5. Simulated protons crossing a stack: in black are the protons hitting the first simulated film, in red the ones hitting the second, in green the third, in blu the fourth and in pink the fifth simulated film. The colour regions on the simulated films are not affected by the shape of the chamber window which in the simulations is larger than the experimental window and rectangular.

the maximum energy protons, which in our case was five. Fewer films can be used if the laser and target conditions can be kept stable.

Once the translation value between the reference system of the experimental and simulated films is known, it was possible to find the spectrum of the protons impinging on the middle of the thickness of the strip active layer along the circles corresponding to the cell spots. From this analysis it was evident that the proton spectra were not always the same for each shot. This was due not only to small movements of the magnet and of the slit holding the target, but also to varying laser pulse characteristics, such as contrast, energy and duration. These are conditions that cannot be simulated with Fluka, but our analysis using the dose deposition on the film stack meant it was possible to approximate the spectrum of the proton beam on the strip and on the cell dish for each shot.

Examples of the simulated proton spectra in the middle of the thickness of the strip active layer, with its accurate composition, are shown in figure 6 (a). The spectra displayed are related to the four cell spots of the same shot with the films shown in figure 3 (b). In all the simulations described in this paper the chamber window was considered to be large and rectangular (unlike the experiment) so there was no problem in simulating the spectrum also for cell spot A. Through these spectra and the beam quality correction factor curve, the first estimates of dose were corrected for the variation of the dose response of the films with proton energy.

The beam quality correction factor, $g_{Q, Q_{0}}$, dependent on the proton energy on the active layer of the strip, includes the relative effectiveness (RE) and the water-to-film stopping power ratio $s_{w, \text { film }}$ as defined in [8], is shown in figure 6 (b). The quality $Q_{0}$ refers to the calibration quality of $29 \mathrm{MeV}$ protons from the Birmingham cyclotron. In the reference the author calculated the $g_{Q, Q_{0}}$ values for EBT films, but it was recalculated 

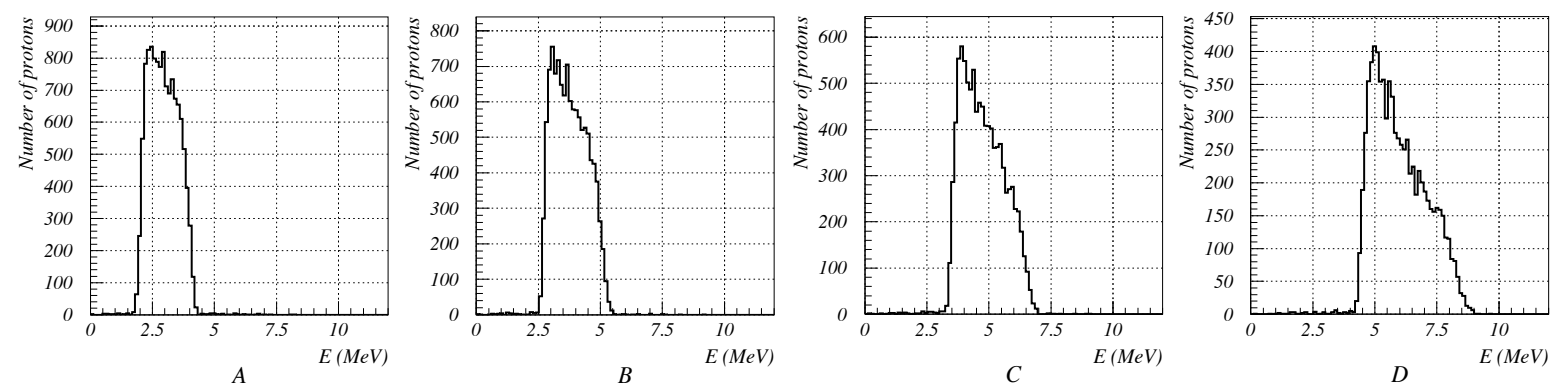

(a)

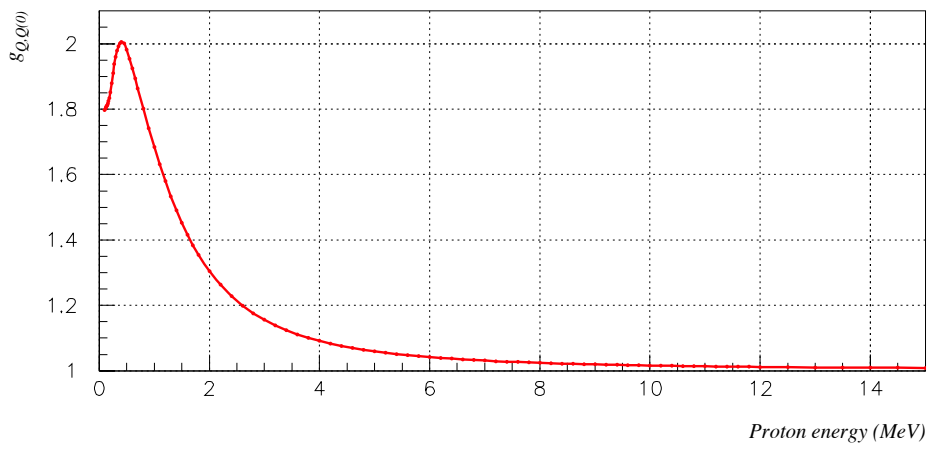

(b)

Figure 6. (a) Extracted spectra of the protons crossing the strip in figure 3 (b) for the four cell spots. The number of simulated protons on the y axis is referred to the case where $10^{7}$ protons were used as an initial beam. (b) Beam quality correction factor, $g_{Q, Q_{0}}$, curve calculated for EBT2 (Daniel Kirby, 2010, private communication).

for EBT2. Knowing that this effect is a direct consequence of the response to the beam energy of the active material in the films, and since the used unlaminated edition of EBT2 films have the same active material as the normal EBT2 films, the $g_{Q, Q_{0}}$ curve can be assumed to be the same. The average beam quality correction factor was calculated for each shot and for each cell spot using the equation 2 :

$$
\bar{g}_{Q, Q_{0 i j}}=\frac{\int_{E_{\operatorname{man}_{i j}}}^{E_{\max _{i j}}} f_{i j}(E) g_{Q, Q_{0}}(E) d E}{\int_{E_{\max _{i j}}}^{E_{\max _{i j}}} f_{i j}(E) d E}
$$

where $i$ represents the shot, $j$ the cell spot, $f(E)$ the proton spectrum in the middle of the thickness of the strip active layer (like the graphs in figure 6 (a)), and $E_{m_{i n} i_{j}}$ and $E_{\text {max }_{i j}}$ respectively the minimum and maximum energy of the proton spectrum in each spot for each shot. The corrected doses, $D s_{i j}$, were subsequently calculated multiplying the correction factor $\bar{g}_{Q, Q_{0_{i j}}}$ by the first estimates of dose, $d_{i j}$, obtained directly from the films. 
2.4. Determination of the correction due to the different doses absorbed by the cells and by the strip active layer

If the cell layer and the active layer of the strip have different equivalent thicknesses or/and are placed at different distances from the source, it is necessary to further correct the doses on the strip to allow for the fact that the absorbed doses in both regions are different. To achieve this the geometry of the cell dish was simulated exactly, differentiating two regions made of water for the cell monolayer and for the overlaying medium, but the active layer of the strip, on this occasion, was simulated made of water and with the equivalent water thickness. For the cell and medium layers the use of water is the usual approximation and it was applied whenever the cells and the medium were simulated. For the active layer of the strip this was due to the fact that the firstlycorrected doses are already dose to water because of the applied calibration. The ratio of the simulated dose in each cell monolayer spot and of the simulated dose in the active layer is the correction. In order to have the real dose absorbed by the cell layer $\left(D c_{i j}\right)$, this ratio $\left(R_{i j}\right)$ has to be multiplied by the first-corrected dose $\left(D s_{i j}\right)$, where $i$ represents the shot and $j$ the cell spot. In our case $R_{i j}$ varied between 0.64 and 1.04 .

\section{Results}

Once the position of the cell spots in the simulation reference system is known, it was possible to score the energy in the middle of their thickness. The graphs in figure 7 are an example of the simulated results for the four cell spots (they are all related to films in figure 3 (b)). Considering both the configurations of the magnet and of the thickness of medium above the cell layer, only for the cell spots B and C (for reasons

explained later), applying a gaussian fit, the values for the mean energy varied from 0.8 to $4.0 \mathrm{MeV}$ for B cell spot and from 2.4 to $5.3 \mathrm{MeV}$ for C. The related $\sigma$ varied from 0.4 to $0.6 \mathrm{MeV}$ for $\mathrm{B}$ and from 0.4 to $0.8 \mathrm{MeV}$ for C.

Having applied both the corrections to the first estimates of dose, it was possible to relate each dose to the mean energy of the protons crossing the corresponding cell spot. Figure 8 shows the corrected doses as a function of the mean beam energy for the cell spots B (in red) and C (in green). The spread of the energies is represented by one standard deviation of the gaussian fit of the spectra. In this graph the different experimental configurations of magnet and amount of medium are well differentiated. The brown data points (only for B cell spot) are due to the configuration where a thick medium layer was placed above the cells and where the magnet and collimator allowed the protons with $3.6 \mathrm{MeV}$ to perpendicularly strike the cell dish. The red (for B cell spot) and green (for $\mathrm{C}$ cell spot) data points are both due to the configuration with lower amount of medium above the cells. In particular the data at lower energies are due to the configuration which allowed the protons with $3.6 \mathrm{MeV}$ to perpendicularly strike the cell dish, and the data at higher energies are due to the configuration which allowed the protons with $6 \mathrm{MeV}$ to perpendicularly strike the cell dish. The average 


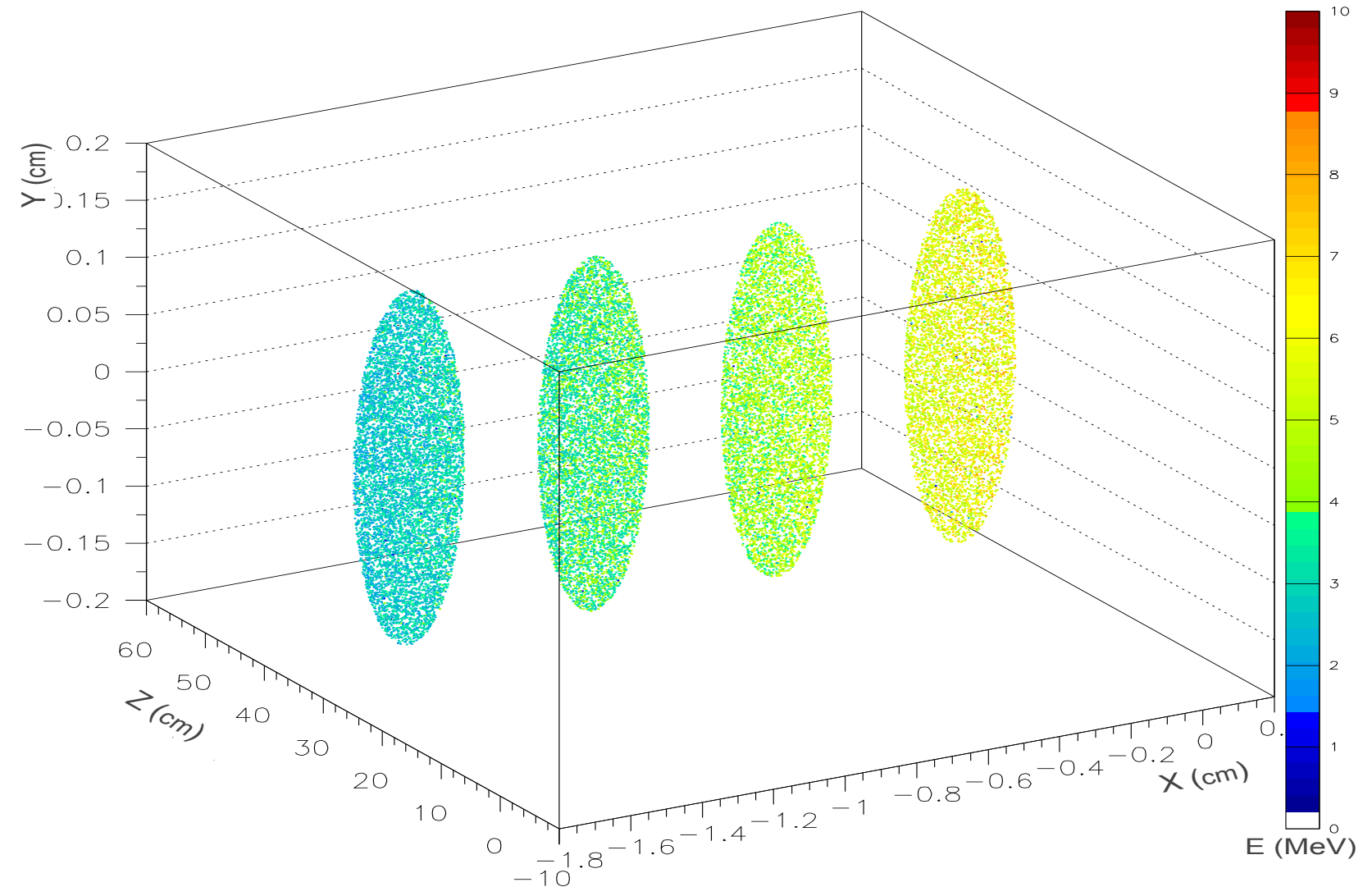

(a)

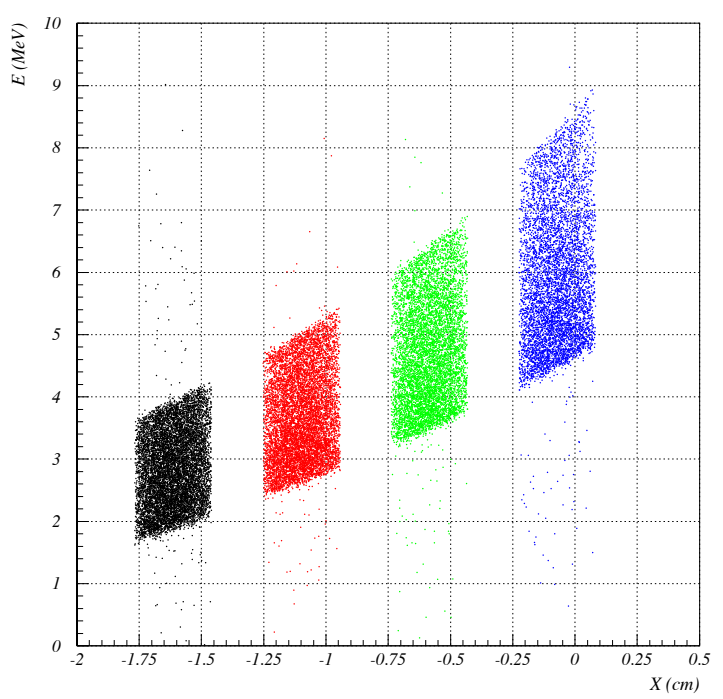

(b)
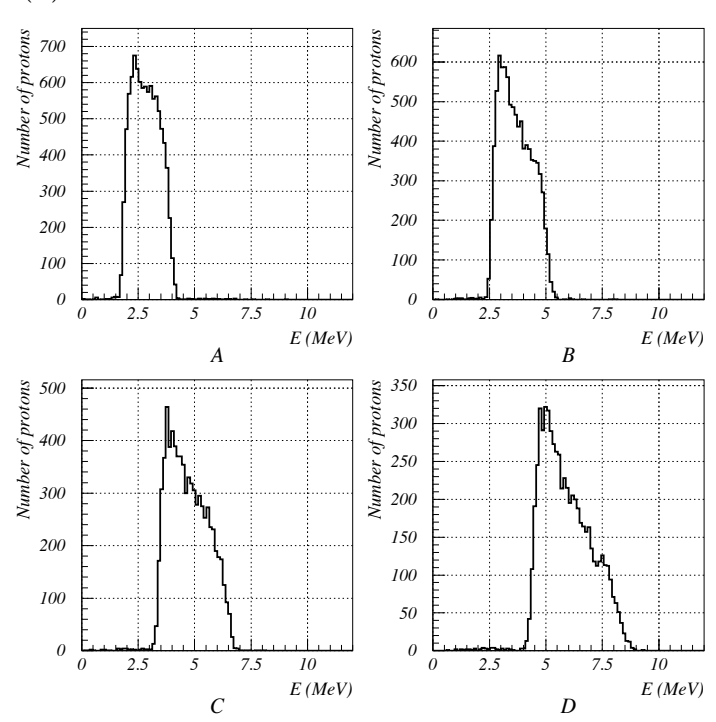

(c)

Figure 7. (a) 4-D graph of the simulated protons in the middle of the thickness of the cell spots. From left to right are A, B, C and D cell spot. (b) Graph of the proton energy versus the horizontal position. The points represent the protons and the different colours indicate the cell spot they are crossing: A (black), B (red), C (green) and D (blue). (c) Extracted proton spectra in the middle of the thickness of the four cell spots. Also in this case the number of simulated protons on the y axis is referred to the case where $10^{7}$ protons were used as an initial beam. All these graphs are related to the films in figure 3 (b). 


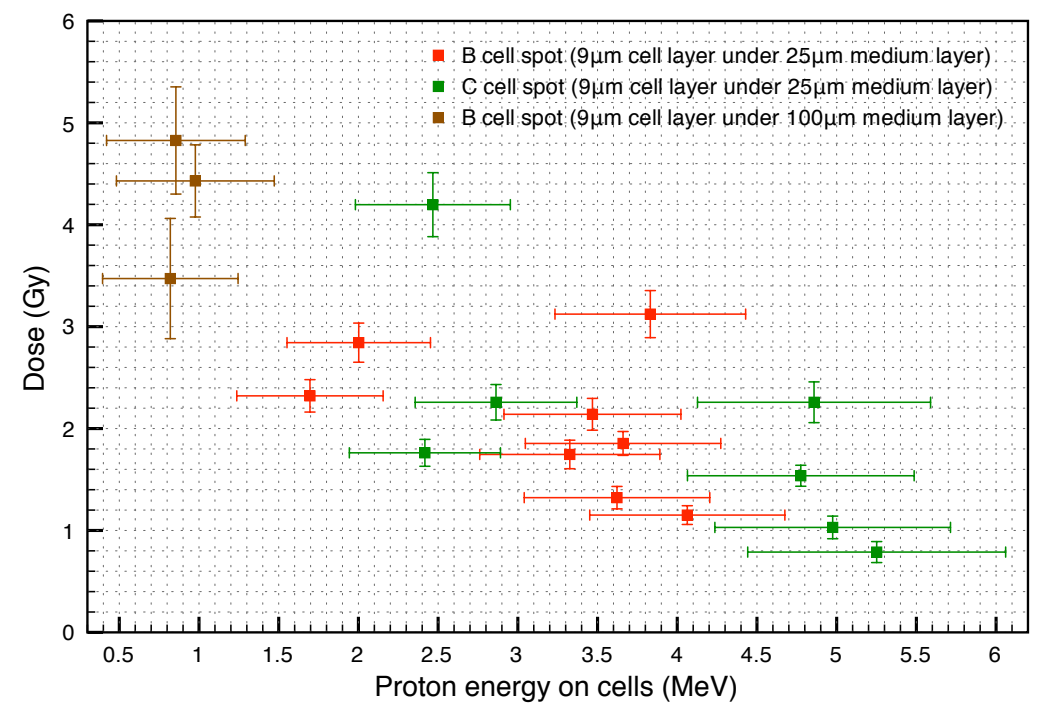

Figure 8. Corrected doses as a function of the energy of the protons crossing the cell spots B and C. The spread used for the energy is one standard deviation of the gaussian fit of the spectra.

energy for brown data points is $(0.88 \pm 0.08) \mathrm{MeV}$, for red data points at low energy is $(1.8 \pm 0.2) \mathrm{MeV}$ and at higher energies is $(3.7 \pm 0.3) \mathrm{MeV}$. The average value for $\mathrm{C}$ data points (green) at low energies is $(2.6 \pm 0.2) \mathrm{MeV}$ and at higher energies is $(5.0 \pm 0.2) \mathrm{MeV}$. The fluctuation of the data around the average energy values in the graph is mainly due to the small deviations of target and laser characteristics already discussed.

Table 1 shows the obtained results for some laser shots. The doses, from the first estimate to the final corrected dose are inserted to show how the corrections affect the data depending on proton energy and on thickness of the medium above the cell layer.

\section{Discussions}

The procedure to obtain the dosimetry deeply involves the use of Gafchromic films and Monte Carlo simulations. The first are useful to have a first estimate of dose on the cells and some information about the position and the energy of the crossing protons (through the dose edges), the second are useful to recreate the experiment and so to discover the spectrum of the protons on the films and on the cell spots, always checking that the information from the experimental and simulated films are in good agreement (through the dose along the films and the dose edge positions).

To summarise: the cell dish is placed in front of a stack of Gafchromic films and behind an energy and charge selection system (figure 2). A film strip can be placed at the same distance from the source as the cell layer (see figure 2 (b)) in order to make a first estimate of dose which is as near as possible to the actual dose absorbed by the cells. This is the approach adopted here, but it is not strictly necessary. In fact, even without using it, applying the second correction described in the paper, the dose 
Dosimetry and spectral analysis of a radiobiological experiment using laser-driven proton beams13

\begin{tabular}{c||c|c|c|c|c} 
Cell spot \#shot & $\Delta z_{\text {medium }}(\mu \mathrm{m})$ & $\overline{E_{p}}(1 \sigma)(\mathrm{MeV})$ & $d_{i j}(\mathrm{~Gy})$ & $D s_{i j}(\mathrm{~Gy})$ & $D c_{i j}(\mathrm{~Gy})$ \\
\hline \hline $\mathrm{B}_{2}$ & 100 & $0.855(0.435)$ & $5.2 \pm 0.3$ & $6.9 \pm 0.3$ & $4.8 \pm 0.5$ \\
\hline $\mathrm{B}_{4}$ & 25 & $1.696(0.459)$ & $1.64 \pm 0.08$ & $2.22 \pm 0.10$ & $2.3 \pm 0.2$ \\
\hline $\mathrm{B}_{6}$ & 25 & $3.831(0.598)$ & $2.76 \pm 0.15$ & $3.06 \pm 0.17$ & $3.1 \pm 0.2$ \\
\hline $\mathrm{B}_{7}$ & 25 & $4.063(0.612)$ & $1.03 \pm 0.06$ & $1.13 \pm 0.07$ & $1.15 \pm 0.09$ \\
\hline $\mathrm{B}_{11}$ & 25 & $3.468(0.555)$ & $1.86 \pm 0.10$ & $2.09 \pm 0.12$ & $2.14 \pm 0.16$ \\
\hline $\mathrm{C}_{4}$ & 25 & $2.417(0.474)$ & $1.36 \pm 0.07$ & $1.67 \pm 0.08$ & $1.76 \pm 0.13$ \\
\hline $\mathrm{C}_{5}$ & 25 & $2.863(0.507)$ & $1.80 \pm 0.10$ & $2.13 \pm 0.11$ & $2.26 \pm 0.17$ \\
\hline $\mathrm{C}_{7}$ & 25 & $4.775(0.711)$ & $1.42 \pm 0.07$ & $1.53 \pm 0.07$ & $1.54 \pm 0.10$ \\
\hline $\mathrm{C}_{9}$ & 25 & $5.251(0.809)$ & $0.75 \pm 0.08$ & $0.80 \pm 0.08$ & $0.79 \pm 0.10$
\end{tabular}

Table 1. Some results obtained for cell spots B and C in different laser shots. The medium thickness $\left(\Delta z_{\text {medium }}\right)$, the mean energy and the sigma (both with a typical uncertainty lower than $1 \%$ ) of the gaussian fit of the spectrum of the protons irradiating the cell layers and the doses are shown for the two cell spots. The doses from the first estimate $\left(d_{i j}\right)$ to the firstly corrected doses $\left(D s_{i j}\right)$ to the final corrected doses $\left(D c_{i j}\right)$ are displayed to illustrate the effect of the two applied corrections.

measured from the first film of the stack will be corrected for the different position and thickness of the cell layer.

The films need then to be scanned (example in figure 3 (b)) and the grey values of the regions corresponding to the cell spots on the strip identified, as well as the dose edge positions on the films of the stack. Using the calibration (equation 1) the grey values are translated to a first estimates of dose, $d_{i j}$ (example in figure 4 ). Using the known initial spectrum of the protons accelerated by the laser, several simulations are performed. One is necessary to find the translation value between the reference system of the simulation and of the experiment and so to locate the cell spots in the simulated experiment. Another is to determine the spectrum of protons crossing the strip in the cell spot regions (examples in figure $6(\mathrm{a})$ ) in order to apply the first correction, $\bar{g}_{Q, Q_{0_{i j}}}$, due to the variation of dose response of the films as a function of proton energy (curve in figure $6(\mathrm{~b})$ ). With this correction the real dose that should have been measured from the active layer of the strip is found: $D s_{i j}=d_{i j} \bar{g}_{Q, Q_{0 i j}}$. The final simulation is necessary to obtain the correction, $R_{i j}$, due to the different thickness and position of the active layer of the strip and of the cell layer, and so to calculate the actual dose absorbed by the cell spots: $D c_{i j}=D s_{i j} R_{i j}$. This simulation is also needed to determine the spectra of the protons crossing the cell spots (examples in figure 7 ).

With regard to the conducted radiobiology experiment, the data shown in figure 9 are those related only to the cell spots $\mathrm{B}$ and $\mathrm{C}$. This is because for $\mathrm{B}$ and $\mathrm{C}$ we usually had: sufficient film area to measure the dose, reasonably high doses and an acceptable energy spread (the spread was in our judgement too large for cell spot D and the doses were too low to add useful survival data). For future experiments, reducing the cell spot diameter would help to decrease the energy spread of the protons crossing them, but would also reduce the number of irradiated cells, so it will need careful consideration. 


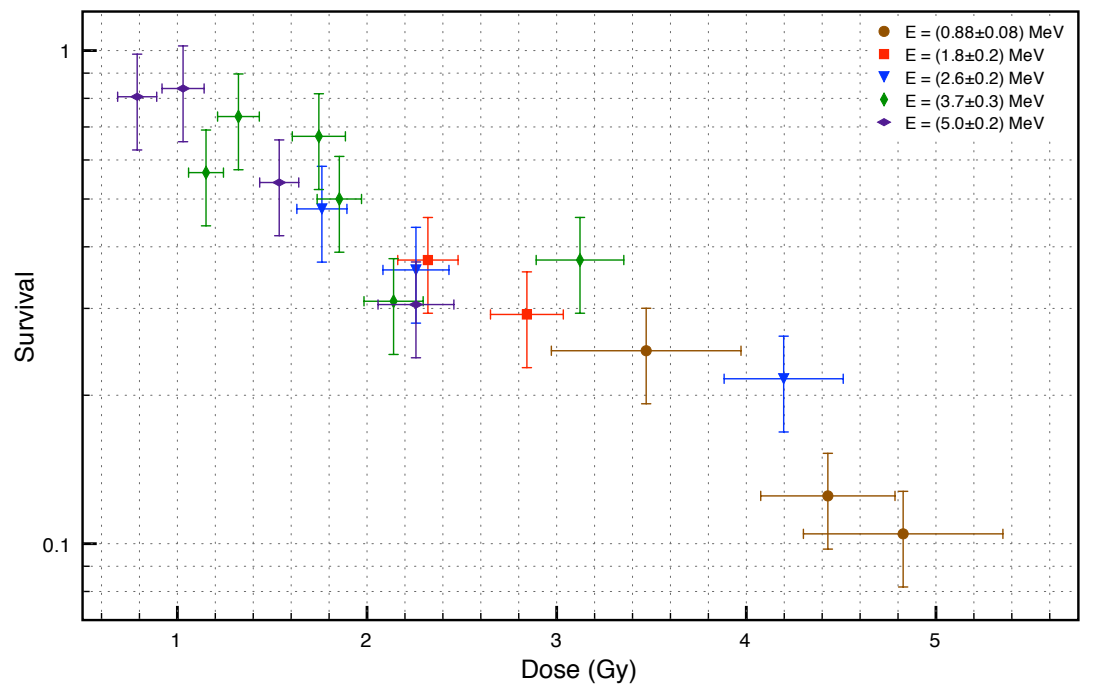

Figure 9. Survival graph of the experimental data for the cell spot B and C. The colour of the points represents the average energy on the cell spot.

The data in figure 9 are differentiated in four different colours according to the average energy of the protons crossing $\mathrm{B}$ and $\mathrm{C}$ cell spots. As already mentioned, in this paper we are not focusing on the radiobiology details of the experiment, but the graph in figure 9 is shown as a demonstration of the good suitability of the method. In any case, there is not sufficient statistics yet to show that different proton energies, which are expected to have different RBE values, create distinct survival curves. We need to increase the number of points with similar energy in order to cover survival from 1 to 0.01 at least, something which is not easy with this kind of single-shot experiment. The same method of handling the cells should then be applied to irradiations non involving laser sources and the comparison between the experiments will allow us to understand whether the high dose rate of the laser driven proton beams might cause different biological consequences from the well known effects of conventionally accelerated beams.

Once the $g_{Q, Q_{0}}$ curve is known, thickness and position of the strip active layer are important contributors to the first correction because they are necessary to have the spectra of the protons in the cell spot positions and so to calculate $\bar{g}_{Q, Q_{0_{i j}}}$. Knowing that the cell layer is a monolayer $9 \mu \mathrm{m}$ thick, the thickness of the medium overlaying the cell layer is the most important contributor of the second correction, affecting the position of the cells and the spectrum of the protons crossing them. The variation in medium thickness does not strongly influence the doses at the highest proton energies, but it is crucial for the lowest energy. In this case, in fact, varying the medium thickness from a thin $(10 \mu \mathrm{m})$ to a thick $(130 \mu \mathrm{m})$ layer, at first the dose increases because the cells are irradiated by protons which are less energetic, reaching a maximum for $\sim 55 \mu \mathrm{m}$ layer: the Bragg peak produced by the proton beam with that initial spectrum is exactly on the cells; then the dose starts decreasing, because the lowest energy protons are stopped in the medium: the Bragg peak is almost in its entirety in the medium, and the cells 
are irradiated by a lower number of protons. The uncertainty in the medium thickness, as well as the cell layer thickness, should, therefore, always be kept at minimum and in particular when working with very low energy protons.

The detail of using very thin Gafchromic films is preferable, but even when it is not possible, the method described here can still be used. In this case the use of the simulations will be more important, because the correction factors to determine the dose absorbed by the cell layer will vary over a much larger range. Also, the first part of the method regarding the dose edge relation between simulated and experimental films will require more attention. In particular at very low energies, the number of dose edges will be smaller (due to the larger thickness of the films) making less space for comparison. If the proton energy is much higher, then, there is no necessity for very thin films.

The dosimetry method and the subsequent spectral analysis are therefore well tested and can be applied in any other similar radiobiological experiment using laser driven proton beams under the condition that the initial laser-driven proton spectrum is reasonably well known. Following all the steps of this method, even for the shots where the proton spectrum is uncertain, it is possible to approximate the spectra on the cells and strip because the comparison between the experimental and simulated dose edges and the dose distribution on the films, provides the necessary additional information. For this reason, it is preferable to use as many films as possible in the stack for each shot, so that it is possible to detect any potential incongruence between the simulated and the experimental dose edges or the presence of peaks in the experimental spectrum.

\section{Acknowledgement}

The experiment was funded by the LIBRA consortium grant from EPSRC, grant $\mathrm{n}^{\circ} \mathrm{EP} / \mathrm{E} 035728 / 1$. A particular acknowledgement goes to David Lewis at International Specialty Products for providing the unlamined samples of EBT2 films, and another one to the Birmingham cyclotron team for their assistance during the irradiations for film calibration.

\section{References}

[1] G Battistoni, S Muraro P R, Sala, F Cerutti, A Ferrari, S Roesler, A Fassò, and J Ranft. The FLUKA code: Description and benchmarking. Proceedings of the Hadronic Shower Simulation Workshop, (Fermilab 6-8 September 2006), M. Albrow, R. Raja eds., AIP Conference Proceeding, 896:31-49, 2007.

[2] S.V. Bulanov and V.S. Khoroshkov. Feasibility of using laser ion accelerators in proton therapy. Plasma Physics Reports, 28(5):453-456, 2002.

[3] E.L. Clark, K. Krushelnick, M. Zepf, FN Beg, M. Tatarakis, A. Machacek, MIK Santala, I. Watts, PA Norreys, and AE Dangor. Energetic heavy-ion and proton generation from ultraintense laser-plasma interactions with solids. Physical Review Letters, 85(8):1654-1657, 2000.

[4] A Fassò, A Ferrari, J Ranft, and P R Sala. FLUKA: a multi-particle transport code, CERN-200510. INFN/TC_05/11, SLAC-R-773, 2005.

[5] F. Fiorini, D. Kirby, S. Green, and DJ Parker. Nuclear activation as a current detector for ion beams produced by a high intensity laser. Radiation Measurements, 45(10):1103-1104, 2010. 
Dosimetry and spectral analysis of a radiobiological experiment using laser-driven proton beams16

[6] M. Hegelich, S. Karsch, G. Pretzler, D. Habs, K. Witte, W. Guenther, M. Allen, A. Blazevic, J. Fuchs, J.C. Gauthier, M. Geissel, P. Audebert, T. Cowan, M. Roth. MeV ion jets from Short-Pulse-Laser interaction with thin foils. Physical Review Letters, 89(8):85002, 2002.

[7] D. Kirby, S. Green, F. Fiorini, D. Parker, L. Romagnani, D. Doria, S. Kar, C. Lewis, M. Borghesi, and H. Palmans. Radiochromic film spectroscopy of laser-accelerated proton beams using the FLUKA code and dosimetry traceable to primary standards. Laser and Particle Beams, 1-9, 2011.

[8] D. Kirby, S. Green, H. Palmans, R. Hugtenburg, C. Wojnecki, and D. Parker. LET dependence of GafChromic films and an ion chamber in low-energy proton dosimetry. Physics in Medicine and Biology, 55:417, 2010.

[9] S.D. Kraft, C. Richter, K. Zeil, M. Baumann, E. Beyreuther, S. Bock, M. Bussmann, TE Cowan, Y. Dammene, W. Enghardt, et al. Dose-dependent biological damage of tumour cells by laseraccelerated proton beams. New Journal of Physics, 12:085003, 2010.

[10] O. Lundh, F. Lindau, A. Persson, C.G. Wahlström, P. McKenna, and D. Batani. Influence of shock waves on laser-driven proton acceleration. Physical Review E, 76(2):26404, 2007.

[11] P. McKenna, K.W.D. Ledingham, T. McCanny, R.P. Singhal, I. Spencer, M.I.K. Santala, F.N. Beg, K. Krushelnick, M. Tatarakis, M.S. Wei. Demonstration of fusion-evaporation and directinteraction nuclear reactions using high-intensity laser-plasma-accelerated ion beams. Physical Review Letters, 91(7):75006, 2003.

[12] M. Murakami, Y. Hishikawa, S. Miyajima, Y. Okazaki, K.L. Sutherland, M. Abe, S.V. Bulanov, H. Daido, T.Z. Esirkepov, J. Koga, et al. Radiotherapy using a laser proton accelerator. First International Symposium on Laser-Driven Relativistic Plasmas Applied to Science, Industry and Medicine AIP Conf. Proc. 1024:275-300, 2008.

[13] T. Dzelzainis, G. Nersisyan, D. Riley, L. Romagnani et al. The TARANIS laser: a multi-Terawatt system for laser-plasma investigations. Laser and Particle Beams, 28:451-461, 2010.

[14] M.D. Abramoff, P.J. Magalhaes, S.J. Ram. Image Processing with ImageJ, Biophotonics International 11, 7, 36-42, 2004

[15] G. Schettino, M. Folkard, K.M. Prise, B. Vojnovic, K.D. Held, and B.D. Michael. Low-dose studies of bystander cell killing with targeted soft X rays. Radiation research, 160(5):505-511, 2003.

[16] P. Thévenaz, U.E. Ruttimann, and M. Unser. A pyramid approach to subpixel registration based on intensity. IEEE Transactions on Image Processing, 7(1):27-41, January 1998.

[17] S.C. Wilks, A.B. Langdon, T.E. Cowan, M. Roth, M. Singh, S. Hatchett, M.H. Key, D. Pennington, A. MacKinnon, and R.A. Snavely. Energetic proton generation in ultra-intense laser-solid interactions. Physics of Plasmas, 8:542, 2001.

[18] J.M. Yang, P. McKenna, K.W.D. Ledingham, T. McCanny, S. Shimizu, L. Robson, R.J. Clarke, D. Neely, P.A. Norreys, M.S. Wei. Nuclear reactions in copper induced by protons from a petawatt laser-foil interaction. Applied physics letters, 84:675, 2004.

[19] A. Yogo, K. Sato, M. Nishikino, M. Mori, T. Teshima, H. Numasaki, M. Murakami, Y. Demizu, S. Akagi, S. Nagayama, et al. Application of laser-accelerated protons to the demonstration of DNA double-strand breaks in human cancer cells. Applied Physics Letters, 94:181502, 2009. 